\title{
The Singularities of the Solutions of the Cauchy Problem
}

\author{
By \\ Yûsaku HAMADA*
}

This paper is concerned with the Cauchy problem of linear partial differential equation with holomorphic coefficients in complex domain.

The Cauchy-Kowalewski theorem asserts that locally there exists a unique holomorphic solution of the Cauchy problem provided that the initial surface is non-characteristic and the initial data are holomorphic.

J. Leray [1] studied in detail the case where the initial surface has characteristic points. He proved the remarkable results that near the characteristic point of the initial surface the solution is ramified around the characteristic surface tangent to the initial surface, and it can be uniformised explicitly (also L. Gårding, T. Kotake and J. Leray [2]).

On the contrary we treat the case where the initial surface is non-characteristic, but the initial data have singularities. In this case, under some conditions, we shall show that the solution has the singularities along the characteristic surfaces issuing from the points of singularity of the initial data. Furthermore we can obtain an explicit representation of the solution.

The purpose of the present paper is to fornulate this situation and to show some results.

Our method relies essentially on the papers of S. Mizohata [4]

Received March 24, 1969.

Communicated by S. Matsuura.

* Department of Mathematics, Kyoto Technical University. 
and [5], in which he constructed the fundamental solution of hyperbolic equation and null solutions. We also use the calculations established by D. Ludwig [3]. Though these analysis were made in the real space, there is no modification to apply them to our problem in the complex domain.

In the next section we describe the precise statement of our results.

I wish to express my sincere gratitude to Professor S. Mizohata for his encouragement.

\section{$\S 1$. Notations and Results}

Let $C^{n}$ be the $n$-dimensional complex space. For the point of $C^{n}$ we use the notation $x=\left(x_{1}, \cdots, x_{n}\right)$ and write $x=\left(x_{1}, x^{\prime}\right), x^{\prime}$ $=\left(x_{2}, \cdots, x_{n}\right)$.

We consider linear partial differential operator of order $m$ with holomorphic coefficients

$$
a\left(x, \frac{\partial}{\partial x}\right)=\sum_{|\alpha| \leq m} a_{\alpha}(x) \frac{\partial^{|\alpha|}}{\partial x_{1}^{\alpha} \alpha^{1 \cdots \partial} x_{n^{n}}^{\alpha}},
$$

where $\alpha=\left(\alpha_{1}, \cdots, \alpha_{n}\right)$ is $n$-tuple of integer $\geqq 0$ with $|\alpha|=\alpha_{1}+\cdots+\alpha_{n}$ and $a_{\alpha}(x)$ are holomorphic in a neighborhood of $x=0$.

We denote by $h(x ; p)$ the homogeneous polynomial in $p$ defined by

$$
\begin{aligned}
h(x ; p) & =h\left(x ; p_{1}, \cdots, p_{n}\right)=\sum_{|a|=m} a_{\alpha}(x) p^{\alpha} \\
& =a_{m, 0}, \cdots, 0(x) p_{1}^{m}+h_{1}\left(x ; p^{\prime}\right) p_{1}^{m-1}+\cdots+h_{m}\left(x ; p^{\prime}\right),
\end{aligned}
$$

where $p^{\alpha}=p_{1^{1} \cdots}^{\alpha} p_{n^{n}}^{\alpha}, p^{\prime}=\left(p_{2}, \cdots, p_{n}\right)$ and $h_{i}\left(x ; p^{\prime}\right)$ are the homogeneous polynomial in $p^{\prime}$ of order $i$.

We shall assume $a_{m, 0}, \cdots, 0,01$ throughout this paper. This means that the surface $x_{1}=0$ is non-characteristic for the differential operator $h\left(x, \frac{\partial}{\partial x}\right)$. 


$$
\begin{gathered}
a\left(x, \frac{\partial}{\partial x}\right) u(x)=0 \\
\frac{\partial^{k} u}{\partial x_{1}^{k}}\left(0, x^{\prime}\right)=w_{k}\left(x^{\prime}\right) \quad \text { for } \quad k=0,1, \cdots, m-1,
\end{gathered}
$$

where the initial condition $w_{k}\left(x^{\prime}\right)$ are holomorphic in a neighborhood of $x^{\prime}=0$ except for $x_{2}=0$.

In solving this problem, we shall make the following assumption (1.2) If $h\left(0 ; p_{1}, 1,0, \cdots, 0\right)=0$, then $h_{p_{1}}\left(0 ; p_{1}, 1,0, \cdots, 0\right) \neq 0$ 。

Now we can construct the characteristic surfaces $K_{1}, \cdots, K_{m}$ issuing from $x_{1}=x_{2}=0$. The surfaces $K_{i}$ are regular and are defined by the equation $\varphi^{(i)}(x)=0\left(\varphi_{x}^{(\varepsilon)} \neq 0 \text { and } \varphi^{(i)}\left(0, x^{\prime}\right)=x_{2}\right)_{0}{ }^{1)}$

Then our statement may be stated as follows

Theorem. Under the assumption (1.2), the Cauchy problem (1.1) has a unique solution $u(x)$ which is analytic in a neighborhood of $x=0$ except for $K_{1} \cup K_{2} \cup \cdots \cup K_{m}$ and has the singularities on $K_{1} \cup K_{2} \cup \cdots \cup K_{m}$.

More precisely, provided that $w_{k}\left(x^{\prime}\right) k=0,1,2, \cdots, m-1$ have at most poles along $x_{2}=0$, the solution $u(x)$ is expressed in the form

$$
u(x)=\sum_{i=1}^{m}\left\{\frac{F^{(i)}(x)}{\left[\varphi^{(i)}(x)\right]^{p_{i}}}+G^{(i)}(x) \log \varphi^{(i)}(x)+H^{(i)}(x)\right\},
$$

where $F^{(i)}(x), G^{(i)}(x)$ and $H^{(i)}(x)$ are holomorphic in a neighborhood of $x=0$ and $p_{i}$ is integer $\geqq 0$.

In the case where at least one of $w_{k}\left(x^{\prime}\right)$ has essential singularities along $x_{2}=0$, the solution $u(x)$ is expressed in the form

$$
u(x)=\sum_{i=1}^{m}\left\{\sum_{k=1}^{\infty} \frac{F_{k}^{(i)}(x)}{\left[\varphi^{(i)}(x)\right]^{k}}+G^{(i)}(x) \log \varphi^{(i)}(x)+H^{(i)}(x)\right\},
$$

where $F_{k}^{(i)}(x), G^{(i)}(x)$ and $H^{(i)}(x)$ are holomorphic in a neighborhood of $x=0$.

Remark. The uniqueness of the solution follows from the Cauchy-Kowalewski theorem.

1) $\varphi^{(i)}(x)$ does not signify the derivative of $\varphi(x)$. 
The proof of this theorem consists of two steps. In the first step, we construct the formal solution satisfying the given conditions. This enables us to calculate actually the integer $p_{i}{ }^{2}{ }^{2} F^{(i)}(x)$ etc. in the expressions (1.3) and (1.4). In the next step we discuss the exactness of the formal solution.

Section 2 contains the construction of the characteristic surfaces $K_{i}$. Section 3 is devoted to the construction of the formal solution and in Section 4 we discuss the exactness of the formal solution and prove the above theorem, in the case where the initial data have at most poles. Section 5 is devoted to proving the theorem in the case where the initial data have essential singularities.

\section{$\S 2$. The Construction of the Characteristic Surfaces}

In this section we shall construct the characteristic surfaces $K_{1}, \cdots, K_{m}$ issuing from $x_{1}=x_{2}=0$.

For this purpose, we consider the first order differential equation

$$
h\left(x, \varphi_{x}(x)\right)=0
$$

with the initial conditions

$$
\varphi\left(0, x^{\prime}\right)=x_{2} .
$$

Thus we have

$$
\varphi_{x_{2}}\left(0, x^{\prime}\right)=1, \quad \varphi_{x_{3}}\left(0, x^{\prime}\right)=0, \cdots, \varphi_{x_{n}}\left(0, x^{\prime}\right)=0 .
$$

By the assumption (1.2), for $|x|$ sufficiently small the equation $h\left(x ; p_{1}, 1,0, \cdots, 0\right)=0$ has $m$ distinct roots $p_{1}=\alpha_{i}(x ; 1,0, \cdots, 0)$ $(i=1,2, \cdots, m)$, where $h\left(x ; p_{1}, \cdots, p_{n}\right)=\prod_{i=1}^{m}\left(p_{1}-\alpha_{i}\left(x ; p_{2}, \cdots, p_{n}\right)\right)^{3)}$ and $\alpha_{i}(x ; 1,0, \cdots, 0)(i=1,2, \cdots, m)$ are holomorphic in a neighborhood of $x=0$. We also write $\alpha_{i}=\alpha_{i}(0 ; 1,0, \cdots, 0)$.

As is well known, $\varphi(x)$ is obtained by solving the associated ordinary differential equations

2) See page 16 .

3) This holds for $\left|p_{2}-1\right|,\left|p_{3}\right|, \cdots,\left|p_{n}\right|$ sufficiently small. 
(2.2)

$$
\left\{\begin{array}{l}
\frac{d x_{i}}{d t}=h_{p_{i}}(x, p) \\
\frac{d p_{i}}{d t}=-h_{x_{i}}(x, p) \quad i=1,2, \cdots, n \\
\frac{d \varphi}{d t}=m h(x, p)
\end{array}\right.
$$

with the initial conditions

$$
\left\{\begin{array}{l}
x_{1}(0)=0, \quad x_{2}(0)=y_{2}, \cdots, x_{n}(0)=y_{n} \\
p_{1}(0)=\alpha_{i}\left(0, y^{\prime} ; 1,0, \cdots, 0\right), \quad p_{2}(0)=1, \quad p_{3}(0)=0, \cdots, p_{n}(0)=0 \\
\varphi(0)=y_{2} .
\end{array}\right.
$$

Let the solution of $(2.2)$ be

$$
\left\{\begin{array}{l}
x_{1}=x_{1}\left(t, y_{2}, \cdots, y_{n}\right) \\
\cdots \cdots \cdots \cdots \cdots \cdots \\
x_{n}=x_{n}\left(t, y_{2}, \cdots, y_{n}\right) \\
\varphi=y_{2}
\end{array}\right.
$$

Then from (2.2) we can easily see that

$$
\left.\frac{D\left(x_{1}, x_{2}, \cdots, x_{n}\right)}{D\left(t, y_{2}, \cdots, y_{n}\right)}\right|_{t=0}=h_{p_{1}}\left(0, y^{\prime} ; \alpha_{i}\left(0, y^{\prime} ; 1,0, \cdots, 0\right), 1,0, \cdots, 0\right) \neq 0
$$

for $\left|y^{\prime}\right|$ sufficiently small.

From the theorem of the implicit function, it follows that

$$
\left\{\begin{array}{c}
t=t\left(x_{1}, \cdots, x_{n}\right) \\
y_{2}=y_{2}\left(x_{1}, \cdots, x_{n}\right) \\
\cdots \cdots \cdots \cdots \cdots \cdots \cdots \\
y_{n}=y_{n}\left(x_{1}, \cdots, x_{n}\right)
\end{array}\right.
$$

where the functions on the right hand side are holomorphic in a neighborhood of $x=0$.

Hence, we have

$$
\varphi=y_{2}=y_{2}\left(x_{1}, \cdots, x_{n}\right)=\varphi^{(i)}\left(x_{1}, \cdots, x_{n}\right) \quad i=1,2, \cdots, m .
$$

Thus, the characteristic surfaces $K_{i}$ issuing from $x_{1}=x_{2}=0$ are given by the equation

$$
\varphi^{(i)}\left(x_{1}, \cdots, x_{n}\right)=0 .
$$

Moreover, from the fact that $\varphi^{(i)}\left(0, x^{\prime}\right)=x_{2}$, we can write 


$$
\varphi^{(i)}(x)=x_{1} \phi^{(i)}(x)+x_{2}, \quad \varphi_{x_{1}}^{(i)}(0)=\alpha_{i} .
$$

Here $\phi^{(i)}(x)$ are holomorphic in a neighborhood of $x=0$.

Since

$$
\varphi_{x_{2}}^{(i)}(0)=1
$$

we conclude that $K_{i}$ are regular surfaces and $\varphi_{x}^{(i)}(x) \neq 0$.

\section{$\S 3$. Construction of Formal Solution}

In this section and the next section, we treat the case where the initial data have at most poles along $x_{2}=0$ on the initial surface $x_{1}=0$.

According to the principle of superposition, it is sufficient to consider the following special initial value problem

$$
a\left(x, \frac{\partial}{\partial x}\right) u(x)=0
$$

and the initial conditions

(3.2) $\frac{\partial^{k} u}{\partial x_{1}{ }^{k}}\left(0, x^{\prime}\right)=0 \quad k \neq h \quad$ and $\quad \frac{\partial^{h} u}{\partial x_{1}{ }^{h}}\left(0, x^{\prime}\right)=(-1)^{l-1}(l-1) ! \frac{w\left(x^{\prime \prime}\right)}{x_{2}^{l}}$

$$
0 \leqslant k \leqslant m-1 \text {, }
$$

where $l$ is the integer $>0$ and $w\left(x^{\prime \prime}\right)$ is holomorphic function of $x^{\prime \prime}=\left(x_{3}, \cdots, x_{n}\right)$.

In solving this problem, we shall make use of the formal solution of (3.1) and (3.2). For this purpose, we introduce the functions $f_{j}(s)(j=-m,-m+1, \cdots)$ satisfying the following relations

$$
\left\{\begin{array}{l}
\frac{d}{d s} f_{j}(s)=f_{j-1}(s) \quad j=-m,-m+1, \ldots \ldots \ldots \ldots \\
f_{-h}(s)=\frac{(-1)^{l-1}(l-1) !}{s^{l}} \\
\quad \ldots \ldots \ldots \ldots \ldots \ldots \ldots \ldots \ldots \ldots \ldots \ldots \ldots \ldots \ldots \ldots \ldots \\
f_{-h+l}(s)=\log s \\
f_{-h+l+\alpha}(s)=\frac{s^{\alpha}}{\alpha !} \log s-\frac{A_{\alpha}}{\alpha !} s^{\alpha} \\
A_{\alpha}=1+\frac{1}{2}+\frac{1}{3}+\cdots+\frac{1}{\alpha} \\
A_{0}=0 .
\end{array}\right.
$$


We assume that with the aid of these functions the solution $u(x)$ has the form

$$
u(x)=\sum_{i=1}^{m}\left\{\sum_{k=0}^{\infty} f_{k}\left(\varphi^{(i)}(x)\right) u_{k}^{(i)}(x)\right\}
$$

Here $u_{k}^{(i)}(x)$ are functions to be determined.

Now, we have the identity

$$
\begin{aligned}
& a\left(x, \frac{\partial}{\partial x}\right)[f(\varphi) u]=f^{(m)}(\varphi) h\left(x, \varphi_{x}\right) u+f^{(m-1)}(\varphi) \\
& \times\left\{\sum_{j=1}^{n} h^{(j)}\left(x, \varphi_{x}\right) \frac{\partial u}{\partial x_{j}}+c_{1}(x) u\right\}+f^{(m-2)}(\varphi) L_{2}[u]+\cdots+f(\varphi) L_{m}[u],
\end{aligned}
$$

where $h^{(j)}(x, \xi)$ implies $\frac{\partial}{\partial \xi_{j}} h(x, \xi)$ and $c_{1}(x)$ is holomorphic function and $L_{p}(p=2,3, \cdots, m)$ are differential operator of order $p$ with holomorphic coefficients.

Let us act the differential operator $a\left(x, \frac{\partial}{\partial x}\right)$ on both sides of (3. 4).

By using the above formula (3.5), we get

$$
\begin{aligned}
& a\left(x, \frac{\partial}{\partial x}\right) u(x)=\sum_{i=1}^{m} \sum_{k=0}^{\infty}\left\{f_{k-m}\left(\varphi^{(i)}\right) h\left(x, \varphi_{x}^{(i)}\right) u_{k}^{(i)}\right. \\
& \quad+f_{k-m+1}\left(\varphi^{(i)}\right)\left(\sum_{j=1}^{n} h^{(j)}\left(x, \varphi_{x}^{(i)}\right) \frac{\partial u_{k}^{(i)}}{\partial x_{j}}+c_{i, 1}(x) u_{k}^{(i)}\right) \\
& \left.\quad+f_{k-m+2}\left(\varphi^{(i)}\right) L_{i, 2}\left[u_{k}^{(i)}\right]+\cdots+f_{k}\left(\varphi^{(i)}\right) L_{i, m}\left[u_{k}^{(i)}\right]\right\} .
\end{aligned}
$$

We recall that $\varphi^{(i)}(x)$ are the solution of the equation $h\left(x, \varphi_{x}^{(i)}(x)\right)=0$.

Thus if we work out formally the coefficients of $f_{j}\left(\varphi^{(i)}\right)$ in (3.6) and set each of them equal to zero, we obtain the sequence of the recursion formulas

(3. 7) $\quad \mathcal{L}_{i}\left[u_{0}^{(i)}\right]=\sum_{j=1}^{n} h^{(j)}\left(x, \varphi_{x}^{(i)}\right) \frac{\partial u_{0}^{(i)}}{\partial x_{j}}+c_{i, 1}(x) u_{0}^{(i)}=0 \quad i=1,2, \cdots, m$

(3. 8) $\mathcal{L}_{i}\left[u_{k}^{(i)}\right]=-\sum_{p=2}^{m} L_{i, p}\left[{\left.u_{k+1-p}^{(i)}\right]} \begin{array}{l}i=1,2, \cdots, m \\ k=1,2, \cdots \cdots\end{array}\right.$

for the determination of $u_{0}^{(i)} u_{1}^{(i)}, \cdots{ }^{4)}$

4) $u_{k}^{(i)}(k<0) \equiv 0$. 
Here we note that the hypothesis (1.2) implies $h^{(1)}\left(x, \varphi_{x}^{(i)}\right) \neq 0$.

In solving (3.7) and (3.8), we find it convenient to give to the first variable $x_{1}$ the special role rather than to use the independent variables $x_{1}, \cdots, x_{n}$. Namely we denote $x_{1}$ by $t$ and $\left(x_{2}, \cdots, x_{n}\right)$ by $\left(x_{1}, \cdots, x_{n}\right)$. We also denote $x^{\prime}$ by $x$ and $x^{\prime \prime}$ by $x^{\prime}$.

Then we can rewrite the equation (3.7) and (3.8) as follows

$$
\begin{aligned}
& \mathcal{L}_{i}\left[u_{0}^{(i)}\right]=\frac{\partial u_{0}^{(i)}}{\partial t}+\sum_{j=1}^{n} a_{i, j}(t, x) \frac{\partial u_{0}^{(i)}}{\partial x_{j}}+c_{i}(t, x) u_{0}^{(i)}=0 \\
& \mathcal{L}_{i}\left[u_{k}^{(i)}\right]=-\sum_{p=2}^{m} L_{i, p}\left[u_{k+1-p}^{(i)}\right] \quad k \geqq 1,
\end{aligned}
$$

Next, Let us turn to a consideration to the initial conditions (3.2). As is easily seen, we have following relations :

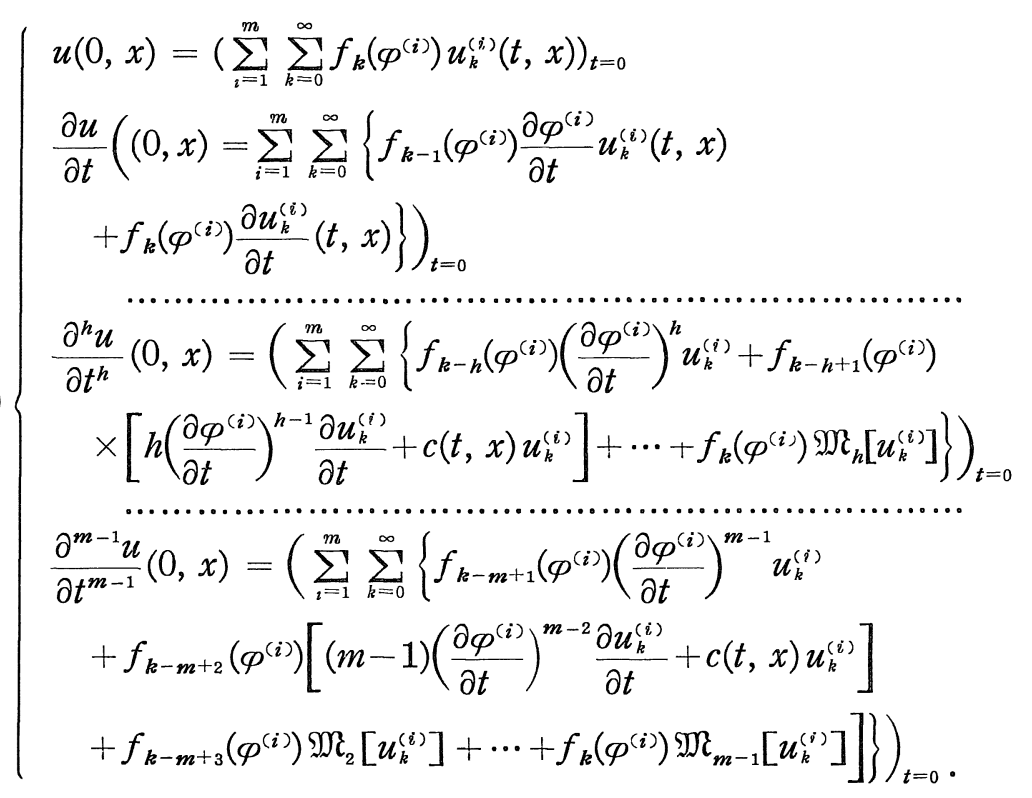

Here, for simplicity in notation, we have denoted by the same notation $\mathfrak{M}_{j},(j=1,2, \cdots, m-1)$ the various differential operators of order $j$ which consist only of the derivatives with respect to $t$. This will not give rise to confusion. From here on until the end of the next section, we use this notation.

Now the right hand side of (3.2) can be expressed in terms of 
$f_{j}\left(x_{1}\right) \quad(j=-h,-h+1, \cdots)$. After doing so, if we compare the coefficients of $f_{j}\left(x_{1}\right)$ in (3.2) wih those of (3.11), then we obtain

$$
\begin{aligned}
& \begin{array}{l}
\sum_{i=1}^{m} u_{0}^{(i)}(0, x)=0 \\
\sum_{i=1}^{m} \alpha_{i}(x) u_{0}^{(i)}(0, x)=0
\end{array} \\
& \begin{array}{c}
\sum_{i=1}^{m}\left[\alpha_{i}(x)\right]^{h} u_{0}^{(i)}(0, x)=w\left(x^{\prime}\right) \\
\ldots \ldots \ldots \ldots \ldots \ldots \ldots \ldots \ldots \ldots \ldots \ldots \ldots \ldots \ldots \ldots \\
\sum_{i=1}^{m}\left[\alpha_{i}(x)\right]^{m-1} u_{0}^{(i)}(0, x)=0,
\end{array}
\end{aligned}
$$

where $\quad \alpha_{i}(x)=\frac{\partial \varphi^{(i)}}{\partial t}(0, x)$.

Generally for $k=1,2, \cdots$, we have

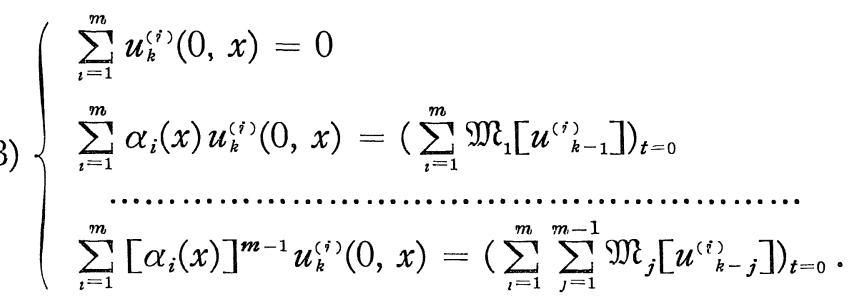

Let

$$
\Delta(x)=\left|\begin{array}{cccc}
1 & 1 & \cdots \cdots \cdots & 1 \\
\alpha_{1}(x) & \alpha_{2}(x) & \cdots \cdots \cdots & \alpha_{m}(x) \\
\vdots & \vdots & & \vdots \\
{\left[\alpha_{1}(x)\right]^{m-1}} & {\left[\alpha_{2}(x)\right]^{m-1}} & \cdots \cdots \cdots & {\left[\alpha_{m}(x)\right]^{m-1}}
\end{array}\right| .
$$

This is Vandermonde's determinant of $\alpha_{1}, \cdots, \alpha_{m}$. As is shown in the preceding section, these are distinct, and hence this determinant does not vanish in a neighborhood of $x=0$. Therefore, from (3.12) and (3.13) we can determine successively the initial data of $u_{k}^{(i)}$ $(k=0,1,2, \cdots)$.

Indeed, for $i=1,2, \cdots, m$ and $k=0,1,2, \cdots$, we get the expressions of the following type

$$
\begin{aligned}
& u_{0}^{(i)}(0, x)=\Delta(h, i, x) w\left(x^{\prime}\right) \\
& u_{k}^{(i)}(0, x)=\sum_{i=1}^{m} \sum_{j=1}^{m-1}\left(\mathfrak{M}_{j}\left[u_{k-j}^{(i)}\right]\right)_{t-0},
\end{aligned}
$$


where $\Delta(h, i, x)$ is the holomorphic function which depends only on $h, i$ and the operator $a\left(x, \frac{\partial}{\partial x}\right)$.

Summarizing the above results, we find that $u_{k}^{(i)}$ are successively determined by the following recursion formulae.

$$
\mathcal{L}_{i}\left[u_{0}^{(i)}\right]=\frac{\partial u_{0}^{(i)}}{\partial t}+\sum_{j=1}^{n} a_{i, j}(t, x) \frac{\partial u_{0}^{(i)}}{\partial x_{j}}+c_{i}(t, x) u_{0}^{(i)}=0
$$

with the initial condition

$$
u_{0}^{(i)}(0, x)=\Delta(h, i, x) w\left(x^{\prime}\right) .
$$

And for $k=1,2, \cdots$

$$
\mathcal{L}_{i}\left[u_{k}^{(i)}\right]=-\sum_{p=2}^{m} L_{i, p}\left[u_{k+1-p}^{(i)}\right]
$$

with the initial condition

$$
u_{k}^{(i)}(0, x)=\sum_{i=1}^{m} \sum_{j=1}^{m-1}\left(\mathfrak{M}_{j}\left[u_{k-j}^{(i)}\right]\right)_{t=0} .
$$

\section{$\S 4$. The Exactness of the Formal Solution}

The Cauchy-Kowalewski theorem guarantees the existence of the holomorphic solution of (3.9), (3.14), (3.10) and (3.15). But in order to show that the formal solution (3.4) is the exact solution of (3.1) and (3.2), we must establish an estimate of $u_{k}^{(i)}$. For this purpose, we use the following propositions 1,2 and 3 due to Mizohata ([4] and [5] especially [5] §5).

In the following we denote $\frac{\partial}{\partial x}$ by $D$.

Proposition 1. Let $a(x)$ and $b(x)$ be two holomorphic functions. We assume

$$
\begin{aligned}
& \left|D^{\nu} a(x)\right| \leqslant \frac{(r+|\nu|) !}{(k \rho)^{|\nu|}} A, \quad k>1 \\
& \left|D^{\nu} b(x)\right| \leqslant \frac{(s+|\nu|) !}{\rho^{|\nu|}} B,
\end{aligned}
$$

where $r$ and $s$ are non negative integer. Then we have the following estimate 


$$
\left|D^{\nu}(a b)(x)\right| \leqslant \frac{(r+s+|\nu|) !}{\rho^{|\nu|}}\left(A B / C_{r}^{r+s}\right) \times \frac{k}{k-1} .
$$

Consider the first order differential operator

$$
\mathcal{L}=\frac{\partial}{\partial t}+\sum_{j=1}^{n} a_{j}(t, x) \frac{\partial}{\partial x_{j}}+c(t, x),
$$

where $a_{j}(t, x)$ and $c(t, x)$ are holomorphic functions, which have the estimates

$$
\left|D_{t, x}^{\nu} a_{j}(t, x)\right| \leqslant \frac{(|\nu|-1) !}{(3 \rho)^{|\nu|-1}} \gamma, \quad|\nu| \geq 1 \quad \mid a_{j}(t, x) ! \leqslant \gamma_{0}
$$

$$
\left|D_{t, x}^{\nu} c(t, x)\right| \leqslant \frac{|\nu| !}{(3 \rho)^{|\nu|}} \gamma, \quad \nu \geq 0 \text {. }
$$

Proposition 2. Under the above condition, we consider the solution $u(t, x)$ of $\mathcal{L}[u]=f(t, x)$ with the initial condition $u(0, x)=0$.

Moreover we assume

$$
\begin{aligned}
& \left|D_{t}^{q} D_{x}^{\nu} f(t, x)\right| \\
& \quad \leqslant \frac{(r+q+|\nu|) !}{\rho^{q+|\nu|}} \exp (\gamma|t|) K(|t|)^{r+q+\mid \nu !}(\gamma n)^{q} A, \quad r \geqq 1
\end{aligned}
$$

then

$$
\begin{aligned}
& \mid D_{t}^{q} D_{x}^{\nu} u(t, x) ! \\
& \quad \leqslant 2 \frac{(r-1+q+|\nu|) !}{\rho^{q+|\nu|}} \exp (\gamma|t|) K(|t|)^{\gamma+q+|\nu|}(\gamma n)^{q} A,
\end{aligned}
$$

where $K(t)=\exp (\gamma n t)(1+\gamma n t)$ and $\gamma$ and $\rho$ are constants such that

$$
\gamma \geqq \min \left(6 \gamma_{0}, 27\right), \quad 0<\rho \leqslant \frac{1}{18} \text {. }
$$

In the case where the initial data do not vanish, we use the following

Proposition 3. Consider the solution $u$ of $\mathcal{L}[u]=0$.

Concerning the initial value $u(0, x)$, we assume

$$
\left|D_{x}^{\nu} u(0, x)\right| \leqslant \frac{(r+|\nu|) !}{\rho^{|\nu|}} A \quad r \geq 0
$$

then

(4. 8) $\left|D_{t}^{q} D_{x}^{\nu} u(t, x)\right| \leqslant 2 \frac{(r+q+|\nu|) !}{\rho^{q+|\nu|}} \exp (\gamma|t|) K(|t|)^{r+q+|\nu|}(\gamma n)^{q} A$, 
where $\gamma$ and $\rho$ are constants satisfying the condition of Proposition 3.

Under the above preparations, let us enter into the estimation of $u_{k}^{(i)}$. Since the coefficients $a_{i, j}(t, x)$ and $c_{i}(t, x)$ in the equations (3.9) and (3.10) are holomorphic, we can assume that there exist constants $\gamma, \gamma_{0}$ and $\rho$ such that

(4.9) $\left|D_{t, x}^{\nu} a_{i, j}(t, x)\right| \leqslant \frac{(|\nu|-1) !}{(3 \rho)^{|\nu|-1}} \gamma, \quad|\nu| \geq 1 ;\left|a_{i, j}(t, x)\right| \leqslant \gamma_{0}$,

(4. $\left.9^{\prime}\right)\left|D_{t, x}^{\nu} c_{i}(t, x)\right| \leqslant \frac{|\nu| !}{(3 \rho)^{|\nu|}} \gamma, \quad \nu \geq 0$

and satisfy (4.6).

In the process in which we estimate successively $u_{k}^{(i)}$, in consideration of the fact that (4.9) and (4.9') hold in (3.9), (3.10), Proposition 2 and 3 , for simplicity we may omit the suffix $i$ of the differential operator $\mathcal{L}_{i}$ and $L_{i, p}$ in (3.9) and (3.10). This will not lead us to confusion. For a moment, we make this convention.

Now, since $w\left(x^{\prime}\right)$ and $\Delta(h, i, x)$ are holomorphic functions, there exist constants $A, B$ and $\rho$ such that

$$
\begin{aligned}
& \left|D_{x}^{\nu} w\left(x^{\prime}\right)\right| \leqslant \frac{|\nu| !}{\rho^{|\nu|}} A \\
& \left|D_{x}^{\nu} \Delta(h, i, x)\right| \leqslant \frac{|\nu| !}{(3 \rho)^{|\nu|}} B,
\end{aligned}
$$

where $A=\max _{\left|x^{\prime}\right| \leqslant \rho}\left|w\left(x^{\prime}\right)\right|$ and $B$ is constant independent of $h, i$.

Then it follows from (4.10), (4.11) and Proposition 1 that

$$
\left|D_{x}^{\nu}\left\{\Delta(h, i, x) w\left(x^{\prime}\right)\right\}\right| \leqslant 2 \frac{|\nu| !}{\rho^{|\nu|}} A \cdot B .
$$

This is an estimate of the initial data of $u_{0}^{(i)}$ and so allows us to apply Proposition 3 to (3.9) and (3.14). Namely, we have

$$
\begin{aligned}
& \left|D_{t}^{q} D_{x}^{\nu} u_{0}^{(i)}(t, x)\right| \\
& \leqslant 2^{2} \frac{(q+|\nu|) !}{\rho^{q+|\nu|}} \exp (\gamma|t|) K(|t|)^{q+|\nu|}(\gamma n)^{q} A B .
\end{aligned}
$$

For general $k \geq 1$, we can establish an estimate of the form 


$$
\begin{aligned}
& \left|D_{t}^{q} D_{x}^{\nu} u_{k}^{(i)}(t, x)\right| \\
& \quad \leqslant c(k) \frac{(k+q+|\nu|) !}{\rho^{k+q+|\nu|}} \exp (\gamma|t|) K(|t|)^{k+q+|\nu|}(\gamma n)^{q},
\end{aligned}
$$

where $c(k)=c_{0}^{k} N A$ and $c_{0}(>1)$ and $N$ denote some constants depending only on the operator $a\left(x, \frac{\partial}{\partial x}\right)$ and

$$
A=\max _{\left|x^{\prime}\right| \leqslant \rho}\left|w\left(x^{\prime}\right)\right|
$$

We shall prove the estimate $(4.14)$ by induction on $k$.

At first we note that by (4.13), (4.14) holds surely for $k=0$. Now let us assume that this estimate (4.14) holds for $k=0,1, \cdots, k$. To establish the validity of (4.14) for $k+1$, we split $u_{k+1}^{(i)}$ into two parts.

$$
u_{k+1}^{(i)}=\widetilde{u_{k+1}^{(i)}}+\widetilde{u_{k+1}^{(i)}} \text {. }
$$

Here $\widetilde{u_{k+1}^{(i)}}$ is defined by the solution of the equation

$$
\mathcal{L}\left[\widetilde{u_{k+1}^{(i)}}\right]=0
$$

with the initial condition

$$
\widetilde{u_{k+1}^{(i)}}(0, x)=\sum_{i=1}^{m}\left\{\sum_{j=1}^{m-1}\left(\mathfrak{M}_{j}\left[{u_{k+1}^{(i)}}_{k+j}\right]\right)_{t=0}\right\}
$$

While $\widetilde{\widetilde{u_{k+1}^{(i)}}}$ is the solution of the equation

$$
\mathcal{L}\left[\widetilde{\widetilde{u_{k+1}^{(i)}}}\right]=-\sum_{p=2}^{m} L_{p}\left[u_{k+2-p}^{(i)}\right]
$$

with the initial condition $\widetilde{\widetilde{u_{k+1}^{(i)}}}(0, x)=0$.

Then we can apply Proposition 3 (resp. Proposition 2) to $\widetilde{u_{k+1}^{(i)}}$ (resp. $\widetilde{u_{k+1}^{(i)}}$ ). At first we consider $\widetilde{u_{k+1}^{(i)}}$. In order to apply Proposition 3 to (4.16) and (4.17), we have to estimate the initial condition (4.17).

Recalling that $\mathfrak{M}_{j}$ represent the differential operators of order $j$, which consist only of the derivatives with respect to $t$, and using (4.14) and Proposition 1, we have 


$$
\begin{gathered}
\left|D_{x}^{\nu} \widetilde{u_{k+1}^{(i)}}(0, x)\right|=\left|\sum_{i=1}^{m} \sum_{j=1}^{m-1} D_{x}^{\nu}\left(\mathfrak{M}_{j}\left[u_{k+1-j}^{(i)}\right]\right)_{t=0}\right| \\
\leqslant m \sum_{j=1}^{m-1} c(k+1-j) M_{j} \frac{(k+1+|\nu|) !}{\rho^{k+1+|\nu|}}(\gamma n)^{j},
\end{gathered}
$$

where $M_{j}(j=1,2, \cdots, m-1)$ are constants depending only on $a\left(x, \frac{\partial}{\partial x}\right)$. Setting $M=\sum_{j=1}^{m-1} M_{j}$, we get easily

$$
\left|D_{x}^{\nu} \widetilde{u_{k+1}^{(i)}}(0, x)\right| \leqslant m M c(k) \frac{(k+1+|\nu|) !}{\rho^{k+1+|\nu|}}(\gamma n)^{m-1},
$$

where $M$ is constant depending only on the differential operator $a\left(x, \frac{\partial}{\partial x}\right)$.

Then Proposition 3 yields the following estimate

$$
\begin{aligned}
& \left|D_{t}^{q} D_{x}^{\nu} \widetilde{u_{k+1}^{(i)}}(t, x)\right| \\
& \leqslant 2 m M c(k) \frac{(k+1+|\nu|+q) !}{\rho^{k+1+|\nu|+q}} \exp (\gamma|t|) K(|t|)^{k+1+|\nu|+q}(\gamma n)^{q+m-1} .
\end{aligned}
$$

Next we turn to the estimate of $\widetilde{\widetilde{u_{k+1}^{(i)}}}$. We observe the derivatives $D_{t}^{q} D_{x}^{\nu}$ of the second member of (4.18). By the assumption on induction, for $2 \leqslant p \leqslant m$ each term is estimated by

$$
\begin{aligned}
& \left|D_{t}^{q} D_{x}^{\nu} L_{p}\left[{u^{(i)}}_{k+2-p}\right]\right| \\
& \leqslant N_{p} c(k+2-p) \frac{(k+2+q+|\nu|) !}{\rho^{k+2+q+|\nu|}} \exp (\gamma|t|) K(|t|)^{k+2+q+|\nu|}(\gamma n)^{q+p}
\end{aligned}
$$

with suitable constants $N_{p}$ determined only by $L_{p}$. Hence, setting $L=\sum_{p=2}^{m} N_{p}$ and summing up over $p$ from 2 to $m$, we get the estimate of the second member of (4.18)

$$
\begin{aligned}
& \left|\sum_{p=2}^{m} D_{t}^{q} D_{x}^{\nu} L_{p}\left[u_{k+2-p}^{(i)}\right]\right| \\
& \leqslant L c(k) \frac{(k+2+q+|\nu|) !}{\rho^{k+2+q+|\nu|}} \exp (\gamma|t|) K(|t|)^{k+2+q+|\nu|}(\gamma n)^{q+m} .
\end{aligned}
$$

Again we note that $L$ depends only on $a\left(x, \frac{\partial}{\partial x}\right)$.

This enables us to apply Proposition 2 to (4.18). We get 


$$
\begin{aligned}
& \left|D_{t}^{q} D_{x}^{\nu} \widetilde{\widetilde{u_{k+1}^{(i)}}}(t, x)\right| \\
& \leqslant 2 L c(k) \frac{(k+1+|\nu|+q) !}{\rho^{k+2+|\nu|+q}} \exp (\gamma|t|) K(|t|)^{k+2+|\nu|+q}(\gamma n)^{q+m} .
\end{aligned}
$$

From the fact that $K(|t|) \leqslant K(1)$ for $|t| \leqslant 1$, we can rewrite the above inequality as follows

$$
\text { (4.20) } \begin{aligned}
& \left|D_{t}^{q} D_{x}^{\nu} \widetilde{\widetilde{u_{k+1}^{(i)}}}(t, x)\right| \\
& \leqslant 2 L \frac{K(1)}{\rho} c(k) \frac{(k+1+|\nu|+q) !}{\rho^{k+1+|\nu|+q}} \exp (\gamma|t|) K(|t|)^{k+1+\mid \nu_{1}+q}(\gamma n)^{q+m} .
\end{aligned}
$$

Let us set

$$
c_{0}=2 \max \left(2 m M(\gamma n)^{m-1}, \quad 2 L \frac{K(1)}{\rho}(\gamma n)^{m}, 1\right) .
$$

Then adding (4.19) and (4.20), we obtain

$$
\begin{aligned}
& \left|D_{t}^{q} D_{x}^{\nu} u_{k+1}^{(i)}(t, x)\right| \\
& \leqslant c_{0} c(k) \frac{(k+1+|\nu|+q) !}{\rho^{k+1+|\nu|+q}} \exp (\gamma|t|) K(|t|)^{k+1+|\nu|+q}(\gamma n)^{q} .
\end{aligned}
$$

Consequently, if we choose for $c(0)$ and $c(k)$ the constants such that

$$
c(0)=2^{2} A B=N A \text { and } c(k)=c_{0}^{k} N A,
$$

we arrive at the desired estimate (4.14) for $k+1$. This completes the induction.

Now, this estimate (4.14), once established, permits us to show that the formal solution (3.4) is the exact solution.

Before doing so, we find it convenient to utilize the original notation $\left(x_{1}, \cdots, x_{n}\right)$ which does not give to the first variable the special role. Then we can rewrite $(4.14)$ as follows

$$
\begin{aligned}
& \left|D_{x}^{\nu} u_{k}^{(i)}(x)\right| \leqslant c_{0}^{k} N A \frac{(k+|\nu|) !}{\rho^{k+|\nu|}} \exp (\gamma \delta) K(\delta)^{k+|\nu|}(\gamma n)^{|\nu|} \\
& =N A \frac{(k+|\nu|) !}{[\rho(\delta)]^{|\nu|}}[c(\delta)]^{k} \exp (\gamma \delta) \quad \text { for } \quad|x|<\delta,
\end{aligned}
$$

where

$$
c(\delta)=\frac{K(\delta) c_{0}}{\rho}, \quad \rho(\delta)=\frac{\rho}{K(\delta) \gamma n}
$$

and

$$
A=\max _{\left|x^{\prime \prime}\right| \leqslant \rho}\left|w\left(x^{\prime \prime}\right)\right|
$$


Now we return to the proof of the exactness of the formal solution (3.4). In order to do so, it suffices to discuss the exactness of the partial sum for any $i$. But it can be written in the form $^{5)}$

$$
\begin{gathered}
\sum_{k=0}^{\infty} f_{k}\left(\varphi^{(i)}(x)\right) u_{k}^{(i)}(x)=\frac{F^{(i)}(x)}{\left[\phi^{(i)}(x)\right]^{l-h}}+\log \varphi^{(i)}(x)\left\{\sum_{k=0}^{\infty} \frac{\left[\phi^{(i)}(x)\right]^{k}}{k !} u_{-h+l+k}^{(i)}(x)\right\} \\
-\sum_{k=1}^{\infty} \frac{A_{k}}{k !}\left[\varphi^{(i)}(x)\right]^{k} u_{-{ }_{-h+l+k}^{(i)}}(x) \quad \text { for } \quad l-h>0
\end{gathered}
$$

and

$$
\begin{gathered}
\sum_{k=0}^{\infty} f_{k}\left(\varphi^{(i)}(x)\right) u_{k}^{(i)}(x)=\log \varphi^{(i)}(x)\left\{\sum_{k=h-l}^{\infty} \frac{\left[\varphi^{(i)}(x)\right]^{k}}{k !} u_{-h+l+k}^{(i)}(x)\right\} \\
-\sum_{k=h-l}^{\infty} \frac{A_{k}}{k !}\left[\varphi^{(i)}(x)\right]^{k} u_{-h+l+k}^{(i)}(x) \quad \text { for } \quad l-h \leqslant 0 .
\end{gathered}
$$

In either case, it is sufficient to discuss the convergence of the series

$$
\sum_{k=\max (|l-l|, 1)}^{\infty} \frac{\left[\phi^{(i)}(x)\right]^{k}}{k !} u_{-h+l+k}^{(i)}(x)
$$

By using (4.21), we have the following inequality for the general term of (4.22)

$$
\left|\frac{\left[\varphi^{(i)}(x)\right]^{k}}{k !} u_{-h+l+k}^{(j)}(x)\right| \leqslant N A \frac{(-h+l+k) !}{k !}[c(\delta)]^{-h+l+k}\left|\phi^{(i)}(x)\right|^{k} .
$$

This shows that (4.22) converges uniformly throughout a neighborhood $\left|\varphi^{(i)}(x)\right|<\frac{1}{c(\delta)}$ of $x=0$. Consequently, we conclude that the formal solution expresses the exact solution in $\{|x|<\varepsilon\}-\bigcup_{i=1}^{m} K_{i}$ for $\varepsilon>0$ sufficiently small. This establishes our theorem in the case where the initial data have at most poles.

\section{$\S 5$. The Case Where the Initial Data Have Essential Singularities}

Since there is no modification necessary for the other cases, we

5) In the case where the initial conditions are given by (3.2), for the integer $p_{i}$ in the expression (1.3) we have $p_{t} \leqslant l-h$. For at least one of $i=1,2, \cdots, m$, we have $p_{\imath}=l-h$. Of course, it may happen that for some $i F^{(\imath)}(x) \equiv 0$. 
shall carry out the discussion merely for the following special case.

$$
a\left(x, \frac{\partial}{\partial x}\right) u(x)=0
$$

and the initial conditions are given by

(5.2) $\left\{\begin{array}{l}u\left(0, x^{\prime}\right) \text { has essential singularities along the surface } x_{2}=0 \\ \text { and } \quad \frac{\partial^{k} u}{\partial x_{1}^{k}}\left(0, x^{\prime}\right)=0 \quad \text { for } 1 \leqslant k \leqslant m-1 .\end{array}\right.$

Now our assumption means that $u\left(0, x^{\prime}\right)$ has Laurent expansion of the form

$$
u\left(0, x^{\prime}\right)=\sum_{l=1}^{\infty} \frac{v_{l}\left(x^{\prime \prime}\right)}{x_{2}^{l}} \quad x^{\prime \prime}=\left(x_{3}, \cdots, x_{n}\right)
$$

where $v_{l}\left(x^{\prime \prime}\right)$ are holomorphic functions in a neighborhood of $x^{\prime \prime}=0$. We again use the principle of superposition, that is, we consider the Cauchy problem

$$
\left\{\begin{array}{l}
a\left(x, \frac{\partial}{\partial x}\right) u_{l}(x)=0 \\
u_{l}\left(0, x^{\prime}\right)=\frac{v_{l}\left(x^{\prime \prime}\right)}{x_{2}^{l}} \\
\frac{\partial^{k} u_{l}}{\partial x_{1}^{k}}\left(0, x^{\prime}\right)=0 \quad \text { for } \quad 1 \leqslant k \leqslant m-1 .
\end{array}\right.
$$

With the aid of the solution $u_{l}(x)$ of this problem, we define $u(x)$ by the sum of the series

$$
u(x)=\sum_{l=1}^{\infty} u_{l}(x)
$$

It is obvious that $u(x)$ satisfies purely formally our conditions (5.1) and (5.2). Therefore if we can show that the series (5.5) converges uniformly on every compact subset of a neighborhood of $x=0$ except for the reunion $K_{1} \cup \cdots \cup K_{m}$ of the characteristic surfaces, we can conclude that $u(x)$ represents the exact solution of our problem.

In the following we proceed to the discussion on the convergence of the series (5.5). 
Now the consideration of $\S \S 3$ and 4 enables us to write $u_{l}(x)$ in the explicit form

$$
\begin{aligned}
u_{l}(x) & =\sum_{i=1}^{m}\left\{\sum_{\alpha=1}^{l}(-1)^{\infty} \frac{(\alpha-1) !}{\left[\varphi^{(i)}(x)\right]^{\infty}} u_{l, l-\alpha}^{(i)}(x)\right. \\
& +\left(\sum_{k=0}^{\infty} \frac{\left[\varphi^{(i)}(x)\right]^{k}}{k !} u_{l, l+k}^{(i)}(x)\right) \log \varphi^{(i)}(x) \\
& \left.-\sum_{k=1}^{\infty} \frac{A_{k}}{k !}\left[\varphi^{(i)}(x)\right]^{k} u_{l, l+k}^{(i)}(x)\right\} .
\end{aligned}
$$

Substituting (5.6) into (5.5), we have the double series

$$
\begin{aligned}
u(x) & =\sum_{i=1}^{m}\left\{\sum _ { l = 1 } ^ { \infty } \left[\sum_{x=1}^{l}(-1)^{\infty} \frac{(\alpha-1) !}{\left[\phi^{(i)}(x)\right]^{\alpha}} u_{l, l-\alpha}^{(i)}(x)\right.\right. \\
& +\left(\sum_{k=1}^{\infty} \frac{\left[\phi^{(i)}(x)\right]^{k}}{k !} u^{(i)}{ }_{l, l+k}(x)\right) \log \varphi^{(i)}(x) \\
& \left.\left.-\sum_{k=1}^{\infty} \frac{A_{k}}{k !}\left[\varphi^{(i)}(x)\right]^{k} u^{(i)}{ }_{l, l+k}(x)\right]\right\} .
\end{aligned}
$$

Since it is sufficient to discuss the sum for any $i$, we may omit the suffix $i$. The estimate of $u_{l, k}$ follows from the result (4.21) of the previous section. In order to do so, we must estimate $v_{l}\left(x^{\prime \prime}\right)$ in the initial data (5.4). Since $v_{l}\left(x^{\prime \prime}\right)$ are the coefficients of Laurent expansion of $u\left(0, x^{\prime}\right)$ it follows from Cauchy's inequality that

$$
\left|D_{x}^{\nu} v_{l}\left(x^{\prime \prime}\right)\right| \leqslant \varepsilon^{l} M(\varepsilon) \frac{|\nu| !}{\rho^{|\nu|}},
$$

where

$$
M(\varepsilon)=\max _{\varepsilon \leqslant\left|x_{2}\right| \leqslant \rho,\left|x^{\prime \prime}\right| \leqslant \rho}\left|u\left(0, x^{\prime}\right)\right| .
$$

By (4.21), taking $\frac{\varepsilon^{l} M(\varepsilon)}{(l-1) !}$ for $A$, we have

$$
\left|u_{l, k}(x)\right| \leqslant N \frac{\varepsilon^{l} M(\varepsilon)}{(l-1) !} k ![c(\delta)]^{k} \exp (\gamma \delta) .
$$

If this estimate is once established, it is easy to examine the convergence of (5.7).

Indeed, at first we consider the partial sum

$$
\sum_{l=1}^{\infty} \frac{(-1)^{l}(l-1) !}{\varphi^{l}} \sum_{k=l}^{\infty} u_{k, k-l} .
$$


By (5.10), after brief computation, we get

$$
\begin{aligned}
& \sum_{k=l}^{\infty}\left|u_{k, k-l}\right| \leqslant \exp (\gamma \delta) N \sum_{k=l}^{\infty} \frac{\varepsilon^{k} M(\varepsilon)}{(k-1) !} c(\delta)^{k-l}(k-l) ! \\
& <\frac{N M(\varepsilon) \varepsilon^{l}}{(l-1) !} \exp (\gamma \delta) \frac{1}{1-\varepsilon c(\delta)} . \quad(\varepsilon c(\delta)<1)
\end{aligned}
$$

Since we have

$$
\sum_{l=1}^{\infty} \frac{(l-1) !}{|\varphi|^{l}} \sum_{k=l}^{\infty}\left|u_{k, k-l}\right|<N \exp (\gamma \delta) \frac{M(\varepsilon)}{1-\varepsilon c(\delta)} \sum_{l=1}^{\infty}\left(\frac{\varepsilon}{|\varphi|}\right)^{l},
$$

we see that (5.11) converges in $|\varphi|>\varepsilon$.

Next we consider the remaining part of (5.7)

$$
\begin{aligned}
& \left\{\sum_{l=1}^{\infty} \sum_{k=0}^{\infty} \frac{[\varphi(x)]^{k}}{k !} u_{l, l+k}(x)\right\} \log \varphi(x) \\
& +\sum_{l=1}^{\infty} \sum_{k=1}^{\infty} \frac{A_{k}}{k !}[\varphi(x)]^{k} u_{l, l+k}(x) .
\end{aligned}
$$

We observe the coefficient of $\log \varphi(x)$ in the first term. From (5.10), after brief computation, it follows that

$$
\begin{gathered}
\sum_{k=0}^{\infty} \frac{|\varphi|^{k}}{k !}\left|u_{l, l+k}\right| \leqslant M(\varepsilon) N \frac{\varepsilon^{l} c(\delta)^{l}}{(l-1) !} \exp (\gamma \delta) \sum_{k=0}^{\infty}(l+k) ! \frac{(c(\delta)|\varphi|)^{k}}{k !} \\
=N \exp (\gamma \delta) M(\varepsilon) l \frac{[\varepsilon c(\delta)]^{l}}{(1-c(\delta)|\varphi|)^{l+1}} .
\end{gathered}
$$

Therefore the double series

$$
\sum_{l=1}^{\infty} \sum_{k=0}^{\infty} \frac{[\varphi(x)]^{k}}{k !} u_{l, l+k}(x)
$$

converges uniformly in $\frac{\varepsilon c(\delta)}{1-c(\delta)|\varphi|}<1$, that is, $|\varphi|<\frac{1-\varepsilon c(\delta)}{c(\delta)}$. This yields that the coefficient of $\log \varphi(x)$ in the first term of (5.11)

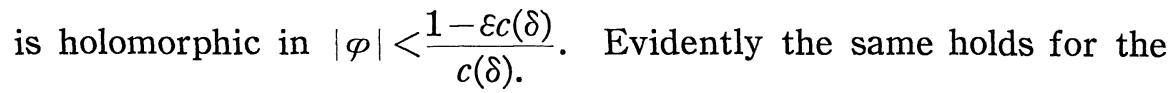
second term of (5.12). Collecting the above results together, we see that (5.7) represents the exact solution in $\varepsilon<|\varphi|<\frac{1-\varepsilon c(\delta)}{c(\delta)}$. Since $\varepsilon(>0)$ is arbitrarily small, we conclude that (5.7) is analytic in $\{|x| \leqslant \sigma\}-\bigcup_{i=1}^{m} K_{i}$ with a suitable positive constant $\sigma$. Further- 
more (5.7) can be rewritten in the form (1.4). This completes the proof of our theorem.

\section{References}

[1] Leray, J., Problème de Cauchy 1, Bull. Soc. Math. France 85 (1957), 389-430.

[2] Gårding, L., T. Kotake et J. Leray, Uniformisation et développement asymptotique de la solution du problème de Cauchy linéaire, a données holomorphes, Bull. Soc. Math. France 92 (1964), 263-361.

[3] Ludwing, D., Exact and asymptotic solutions of the Cauchy problem, Comm. Pure Appl. Math. 13 (1960), 473-508.

[4] Mizohata, S., Solutions nulles et solutions non analytiques, J. Math. Kyoto Univ. $1(1961 / 62), 271-302$.

[5] - Analyticity of the fundamental solutions of hyperbolic systems, J. Math. Kyoto Univ. 1 (1961/62), 327-355. 